\title{
Evolution and Human Choice over Time
}

\author{
Alan R. Rogers*广
}

1997

\section{The connection between evolution and economics}

In economics, equilibria are often found by equating two versions of the marginal rate of substitution (MRS). For example, my MRS in preferences (the ratio at which I am "just willing" to exchange two goods) should equal the MRS in exchange (the ratio at which I can exchange them in the market). Otherwise, I would have reason to sell one good and buy the other. At equilibrium (as shown in figure 1) these two versions of the MRS must be equal.

This analysis is also familiar to evolutionary ecologists, as shown in figure 2 . There, the indifference curves are replaced by fitness isograms, which connect points of equal Darwinian fitness. ${ }^{1}$ In place of a budget constraint, ecologists study a variety of other constraints. The principle, however, is the same: equilibrium occurs at the point where the two curves have equal slope.

These two forms of analysis are connected by something deeper than analogy. They are connected by a third equilibrium principle, which was first described by Hansson and Stuart [12]. These authors define the MRS in fitness as the ratio at which two goods can be exchanged without affecting Darwinian fitness. Thus, the MRS in fitness measures the absolute slope of the dotted lines in figure 2 . The new equilibrium principle asserts that, at evolutionary equilibrium, the MRS in fitness must equal that in preferences. A simple proof of this principle is shown in figure 3.

The new equilibrium principle adds an additional equation to the arsenal of economics. The MRS in preferences must now equal that in fitness as well as those in exchange and production. If the hypothesis of evolutionary equilibrium turns out to be useful, then this should allow a more powerful theory of economics.

*Research Centre, King's College, Cambridge CB2 1ST, U.K. Present address: Dept. of Anthropology, University of Utah, Salt Lake City, UT 84112, U.S.A.

${ }^{\dagger}$ Published as pp. 231-252 in Characterizing Human Psychological Adaptations, edited by G. Bock and G. Cardew. CIBA Foundation Symposium 208. John Wiley and Sons.

${ }^{1}$ In models with discrete generations, Darwinian fitness is the conditionally expected number of an individual's offspring, given its genotype. In models with overlapping generations, fitness is measured by R. A. Fisher's [8] "Malthusian parameter," which measures the asymptotic rate of exponential increase in the numbers of one's descendants [2]. 


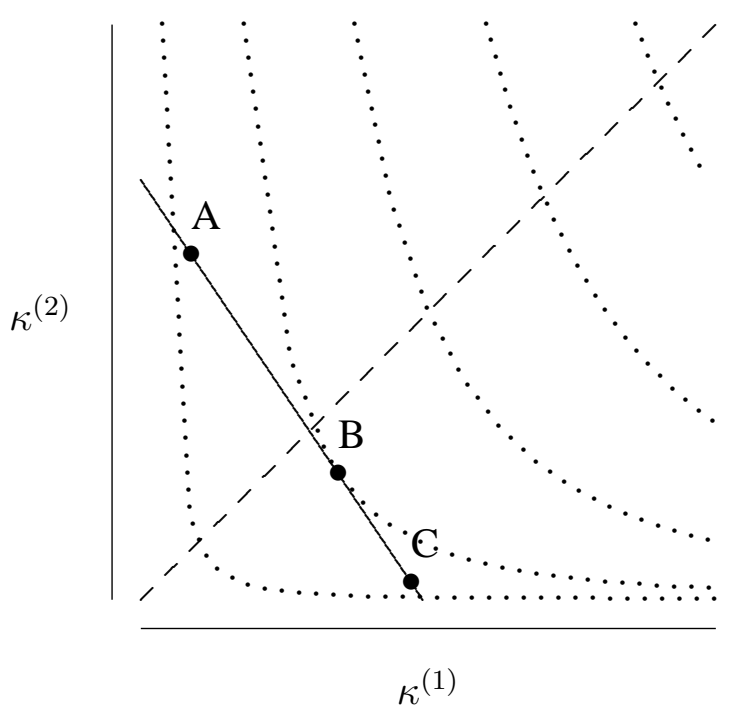

Figure 1: The Indifference Diagram of Economics

An individual consumes a quantity $\kappa^{(1)}$ of good 1 , and $\kappa^{(2)}$ of good 2. The dotted indifference curves connect consumption bundles to which he is indifferent. By buying or selling, the consumer moves left or right along the solid budget line. Utility is maximized at the point where the two lines have equal slope, or in other words, at the point where $M R S_{P}=M R S_{E}$.

\section{Application to time preference}

Suppose that, in figure 1, good 1 refers to food that is consumed today, and good 2 to food that is consumed $\tau$ time units later. With this interpretation, the figure describes preferences regarding different paths of consumption over time, or in other words, time preference. In a recent paper [15], I developed an evolutionary theory of time preference using the methods outlined above. That paper simplified the problem by assuming that changes in consumption affect fitness solely via their effect on survival. Here, I extend that analysis to incorporate effects on fertility as well.

The analysis proceeds by deriving an expression for the MRS in fitness, and setting this equal to well-known expressions for the MRS in preferences and in exchange. I begin with a series of definitions.

\subsection{Definitions}

The MRS in preferences between immediate and delayed consumption is defined by

$$
M R S_{P} \equiv-\left.\frac{d \kappa^{(2)}}{d \kappa^{(1)}}\right|_{U \text { constant }}
$$

where the derivative is taken along a line of constant utility $U$, i.e. an indifference curve. The MRS in preferences is often measured by $\theta$, the marginal rate of time preference (MRTP), which defined 


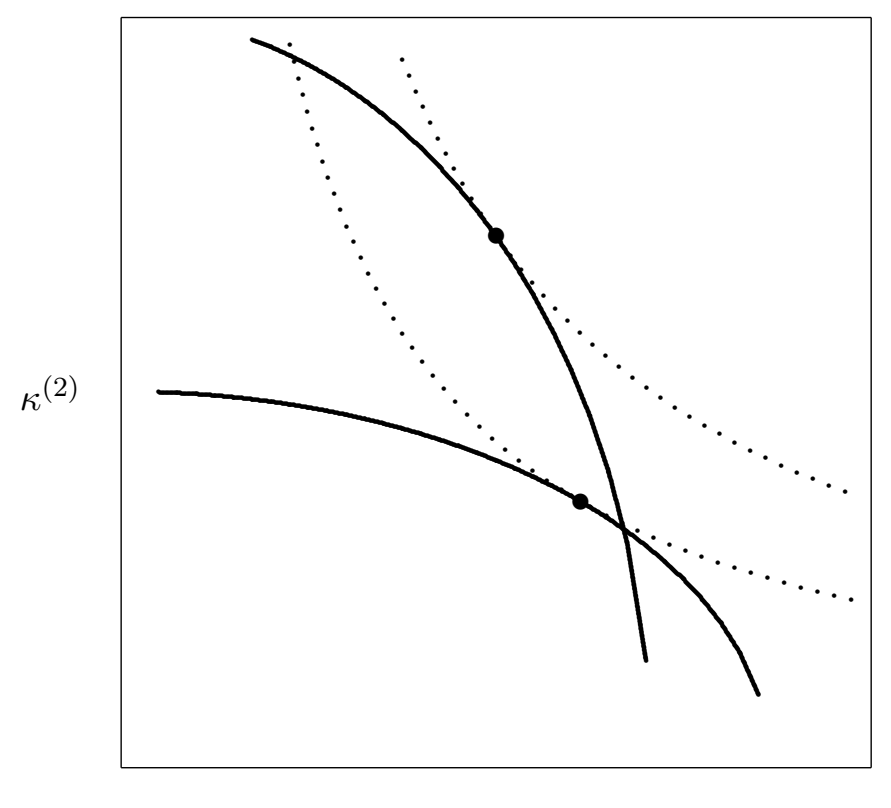

$\kappa^{(1)}$

Figure 2: Constrained Optimization in Evolution

Darwinian fitness increases with increasing values of characters $\kappa^{(1)}$ and $\kappa^{(2)}$, and the dotted fitness isograms connect points of equal fitness. The two solid constraint lines illustrate two different hypotheses about which combinations of $\kappa^{(1)}$ and $\kappa^{(2)}$ are feasible. For any assumed constraint, the evolutionary problem is to choose the point on the constraint line that maximizes fitness. This constrained optimum occurs where the constraint line and fitness isogram have equal slope, i.e. where $M R S_{C}=M R S_{F}$. 


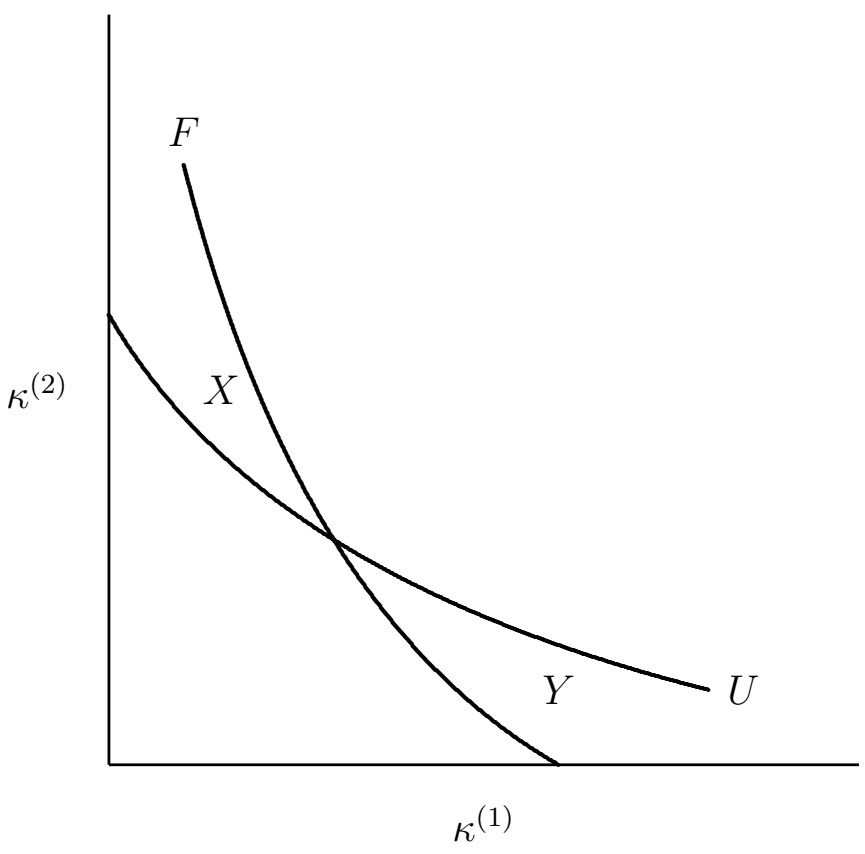

Figure 3: Why the MRS in fitness equals that in preferences at evolutionary equilibrium Fitness and utility each depend on consumption of commodities $\kappa^{(1)}$ and $\kappa^{(2)}$. If the MRS in fitness did not equal that in preferences, then the isograms of the fitness function $F$ would cross those of the utility function $U$, as shown in the figure. There would then exist consumption bundles, $X$ and $Y$, such that $X$ is preferred to $Y$ although $Y$ confers the higher fitness. This preference ordering cannot be evolutionarily stable because a mutation that reversed the preference between $X$ and $Y$ would be favored by selection. 
by

$$
M R S_{P} \equiv e^{\theta \tau}
$$

where as before $\tau$ is the time that elapses between $\kappa^{(1)}$ and $\kappa^{(2)}$. The MRS in exchange between present and future consumption is the ratio at which present and future consumption can be exchanged by borrowing and lending. It is related to the interest rate $i$ by

$$
\operatorname{MRS}_{E} \equiv-\left.\frac{d \kappa^{(2)}}{\left.d \kappa^{(1)}\right)}\right|_{W \text { constant }} \equiv e^{i \tau}
$$

where $W$ is wealth and the derivative is taken along a line of constant wealth, that is, along the solid market line in figure 1 . The MRS in fitness is defined by

$$
M R S_{F} \equiv-\left.\frac{d \kappa^{(2)}}{d \kappa^{(1)}}\right|_{F \text { constant }}
$$

where the derivative is taken along a line of constant fitness $F$. In equilibrium, all these versions of the MRS must be equal.

\subsection{Finding the MRS in fitness}

The evolutionary theory of time preference is complicated by the possibility that the returns from an investment may increase the Darwinian fitness of the investor's daughter (or other relative) rather than that of the investor herself. This makes it necessary to use the evolutionary theory of "kin selection," which deals with interactions between relatives $[9,10]$.

The particular model used here was developed in another context [14], and its application to the economic problem of time preference is discussed elsewhere [15]. Rather than repeat that material here, I shall simply state the relevant results.

\subsubsection{Results from the evolutionary theory of kin selection}

The theory supposes that one individual (the donor or investor) undertakes an investment that has an immediate effect on himself, but a delayed effect on a second individual (the recipient). The donor and recipient may or may not be the same individual. The donor undertakes his action at age $x^{(1)}$, and the recipient is affected after $\tau$ time units, when the recipient's age is $x^{(2)}$. This interaction changes from $P^{(1)}$ to $P^{(1)}+\Delta P^{(1)}$ the donor's probability of surviving from age $x^{(1)}$ to $x^{(1)}+d x$. The donor's fertility during this same interval is changed from $m^{(1)}$ to $m^{(1)}+\Delta m^{(1)}$. Similarly, the interaction changes from $P^{(2)}$ to $P^{(2)}+\Delta P^{(2)}$ the recipient's probability of surviving from age $x^{(2)}$ to $x^{(2)}+d x$. The recipient's fertility during this interval is changed from $m^{(2)}$ to $m^{(2)}+\Delta m^{(2)}$.

The effect of this interaction on Darwinian fitness are summarized in table 1, which is adapted from table 1 of [14]. Unlike the table used in my earlier work on time preference [15], this one includes effects on fertility as well as on mortality. In the table, $r$ denotes the coefficient of relationship between donor and recipient, ${ }^{2}$ the subscripts $D$ and $R$ indicate the sex of the donor and of

\footnotetext{
${ }^{2}$ Wright's coefficient of relationship [5, pp. 69, 137-138] can be interpreted as the fraction of their genes that two individuals can expect to hold in common. It equals 1 if the donor and recipient are the same individual, 1/2 if the recipient is an offspring, 1/4 if a grandchild, and so forth.
} 
Table 1: How Changes in Fertility and Mortality Affect Fitness

\begin{tabular}{lcccc}
\hline \hline $\begin{array}{l}\text { Effect } \\
\text { on }\end{array}$ & $\begin{array}{c}\text { Additive } \\
\text { change }\end{array}$ & $\begin{array}{c}\text { Reproductive } \\
\text { value }\end{array}$ & $\begin{array}{c}\text { Discount } \\
\text { factor }\end{array}$ & $\begin{array}{c}\text { Relationship } \\
\text { to donor }\end{array}$ \\
\hline $\begin{array}{c}\text { Donor } \\
\text { A. fert. }\end{array}$ & $\Delta m^{(1)}$ & 1 & $e^{-\rho x^{(1)}}$ & 1 \\
B. mort. & $\Delta P^{(1)}$ & $v^{(1)}$ & $e^{-\rho\left(x^{(1)}+d x\right)}$ & 1 \\
Recipient & & & & \\
C. fert. & $\Delta m^{(2)}$ & 1 & $e^{-\rho\left(x^{(1)}+\tau\right)}$ & $r$ \\
D. mort. & $\Delta P^{(2)}$ & $v^{(2)}$ & $e^{-\rho\left(x^{(1)}+\tau+d x\right)}$ & $r$ \\
\hline
\end{tabular}

Notes: The altruist allele will increase (decrease) in frequency if the sum of row products is positive (negative). The notation is defined in the text. For simplicity, I assume that the sex ratio at birth is unity, that effects on fertility are brief, that these effects are small enough that second-order terms in $\Delta m$ and $\Delta P$ can be ignored, and that a single recipient is affected by each altruistic act. Source: Rogers [14, Table 1]

the recipient, and $v$ denotes the reproductive value (R. A. Fisher, 1958). It is defined by

$$
v \equiv \frac{\sum_{y=x}^{\infty} e^{-\rho y} l_{g}(y) m_{g}(y)}{e^{-\rho x} l_{g}(x)}
$$

where $\rho$ is the rate of population growth, $l_{g}(y)$ the probability of living to age $y, m_{g}(y)$ the expected number of offspring produced at that age, and the subscript $g$ indicates the individual's sex. The reproductive value can be interpreted as the expected present value of an individual's future contributions to the gene pool.

A gene that encourages the donor to undertake this action will be favored by natural selection if the sum of the row-products in table 1 is positive, or disfavored if that sum is negative.

\subsubsection{The MRS in fitness}

An interaction is selectively neutral-having no effect on fitness-if the sum of row-products in table 1 is zero, i.e. if

$$
\begin{aligned}
0=\Delta m^{(1)} e^{-\rho x^{(1)}}+\Delta P^{(1)} v^{(1)} e^{-\rho x^{(1)}} & \\
& +\Delta m^{(2)} e^{-\rho\left(x^{(1)}+\tau\right)} r+\Delta P^{(2)} v^{(2)} e^{-\rho\left(x^{(1)}+\tau\right)} r
\end{aligned}
$$

Here, I have assumed that effects on mortality are brief so that $d x \approx 0$. When this equation holds, the interaction (or investment) described above moves us along a fitness isogram. Thus, the equation holds the key to the slope of this isogram, the MRS in fitness. But before proceeding, it will be useful to recast the equation in terms of changes in consumption.

I now assume that fertility and mortality are both differentiable functions of consumption.

$$
\begin{aligned}
& P=P(x, \kappa) \\
& m=m(x, \kappa)
\end{aligned}
$$


where $\kappa$ is consumption at age $x$. Furthermore, I assume that the fertility and mortality effects in the table were produced by changes in consumption. Specifically, the donor's consumption at age $x^{(1)}$ changed from $\kappa^{(1)}$ to $\kappa^{(1)}+\Delta \kappa^{(1)}$, while that of the recipient changed from $\kappa^{(2)}$ to $\kappa^{(2)}+\Delta \kappa^{(2)}$. If these changes are small, then the fertility and mortality effects are

$$
\begin{aligned}
\Delta P & \approx \Delta \kappa P_{\kappa}(x) \\
\Delta m & \approx \Delta \kappa m_{\kappa}(x)
\end{aligned}
$$

where $P_{\kappa} \equiv \partial P(x, \kappa) / \partial \kappa$ is the marginal effect of consumption on survival, and $m_{\kappa} \equiv \partial m(x, \kappa) / \partial \kappa$ the marginal effect on fertility. Substituting these into equation 5 and rearranging gives the MRS in fitness,

$$
M R S_{F} \equiv-\frac{\Delta \kappa^{(2)}}{\Delta \kappa^{(1)}}=\left(\frac{e^{\rho \tau}}{r}\right)\left(\frac{m_{\kappa}^{(1)}+P_{\kappa}^{(1)} v^{(1)}}{m_{\kappa}^{(2)}+P_{\kappa}^{(2)} v^{(2)}}\right)
$$

This generalizes Eqn. 7 of my earlier paper [15], which excluded the marginal effect of consumption on fertility.

\subsubsection{The long-term real rate of interest}

The long-term interest rate is found by setting setting

$$
M R S_{F}=e^{i \tau}
$$

where $i$ is the interest rate over delay $\tau$. This procedure equates the MRS in fitness (the left-hand side of the equation) with that in exchange (the right-hand side), and is justified as follows. The argument in figure 3 shows that, in evolutionary equilibrium, the MRS in fitness must equal that in preferences. Furthermore, in market equilibrium the MRS in preferences must equal that in exchange. In studying equation 9, we are examining the implications of the hypothesis that both equilibrium assumptions hold true.

As in my previous paper on time preference, I concentrate on intergenerational investments in which the investment benefits the investor's daughter after exactly one generation. By assumption, the mother and daughter are affected at the same age, so that the two reproductive values in $M R S_{F}$ are equal. In stationary equilibrium, the mother and daughter will also have equal wealth at this common age, so that the marginal effects of consumption on their fertility and survival are equal as well. Consequently, the right-most fraction in equation 8 equals unity, and $M R S_{F}=e^{\rho \tau} / r$, where $r=1 / 2$ (since the two individuals are mother and daughter), and $\tau$ equals the generation length, $T$. Equation 9 becomes $2 e^{\rho T}=e^{i T}$, or

$$
i=(\ln 2) / T+\rho
$$

The relevant rate of population growth is not the current one, but some sort of average rate over recent evolutionary history. Since evolutionary changes are usually slow, the last couple of centuries of rapid growth have probably had no large effect. Prior to that, $\rho$ must on average have been near zero. Thus, equation 10 suggests that $i \approx(\ln 2) / T$. The generation time $T$ is usually a little less 
than 30 years in human populations. For example, $T=28.9$ in the 1906 population of Taiwan [11]. Thus, if $\rho \approx 0$, selection should favor long-term interest rates that average $(\ln 2) / 28.9=0.024$ per year, in reasonable agreement with observation.

These results are identical to those of my earlier paper on time preference [15, Eqn. 12], and extend those results to the more general context in which selection acts via fertility as well as mortality.

\subsection{Diminishing marginal returns to consumption}

I now introduce the standard assumptions of economic analysis: that consumption helps in some sense and that each successive unit of consumption helps less than the last. In the present context, this will mean both that $m(x)$ and $P(x)$ each increase with consumption, and also that marginal effects decline as consumption increases.

Although these assumptions are unremarkable in economics, they may seem problematic here. Eating too much can be bad for you, and animals on restricted diets often seem to live longer than those with unrestricted access to food [7, Sec. 10.3.1]. Yet this is no real cause for skepticism: food is just one of many consumer goods, and wealthy people do live longer than poor ones.

To capture the diminishing marginal effect of consumption, I will assume that

$$
\begin{aligned}
m(x, \kappa) & \equiv m^{*}(x) \kappa^{\alpha} \\
P(x, \kappa) & \equiv P *(x) \kappa^{\beta}
\end{aligned}
$$

where $0<\alpha, \beta<1$, and attention must be restricted to to parameter values such that $P$ stays within the interval $[0,1]$. Here $m^{*}(x)$ and $P *(x)$ are, respectively, the fertility and survival probability of a "standard" individual of age $x$ - one who consumes a single unit of resource.

To justify this particular formulation, I appeal to the data in figure 4. There, the vertical axis measures the variation of age-specific fertility across populations, and the horizontal axis measures mean age-specific fertility. The graph shows that fertility is most variable at age classes where fertility is high. At least some of this variation must reflect variation in consumption. Thus, it is sensible to build a model in which the effect of consumption is greatest on age classes with high fertility. ${ }^{3}$

Marginal fertility and survival become

$$
\begin{aligned}
m_{\kappa} & =\frac{\alpha}{\kappa} m(x, \kappa) \\
P_{\kappa} & =\frac{\beta}{\kappa} P(x, \kappa)
\end{aligned}
$$

and the $M R S$ in fitness is

$$
M R S_{F}=\left(\frac{e^{\rho \tau}}{r}\right)\left(\frac{\gamma m^{(1)}+(1-\gamma) P^{(1)} v^{(1)}}{\gamma m^{(2)}+(1-\gamma) P^{(2)} v^{(2)}}\right)\left(\frac{\kappa^{(2)}}{\kappa^{(1)}}\right)
$$

where $\gamma \equiv \alpha /(\alpha+\beta)$ measures the importance of marginal fertility relative to marginal survival.

\footnotetext{
${ }^{3}$ I need to repeat this exercise with survival data.
} 


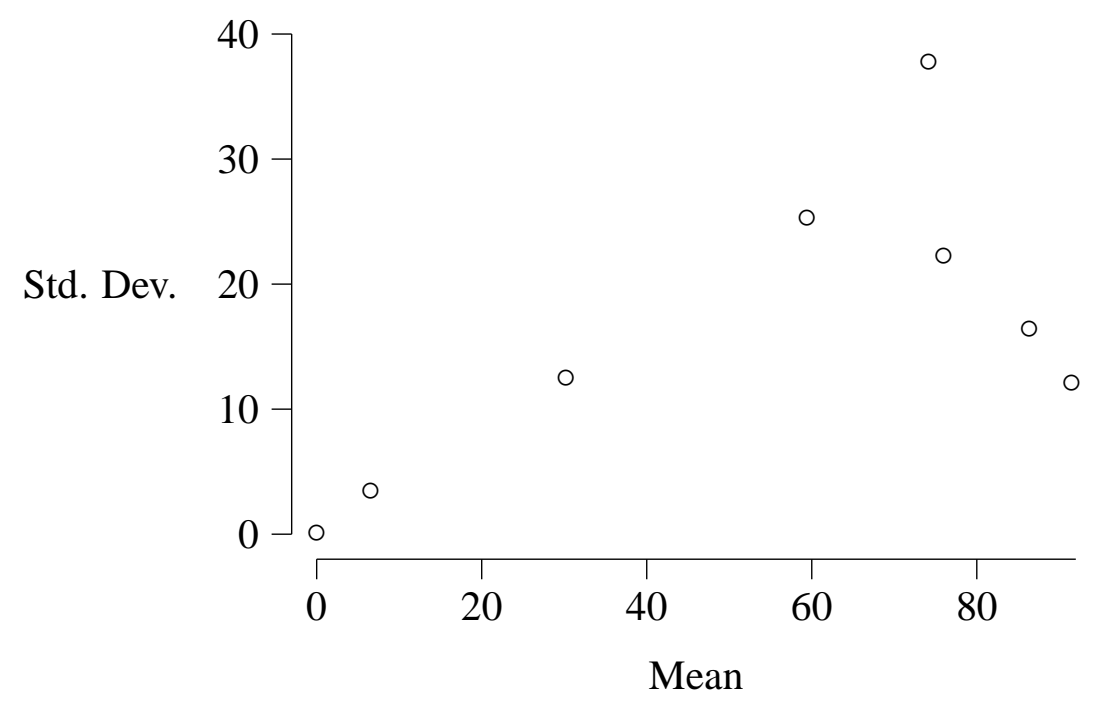

Figure 4: Mean and Standard Deviation of Age-specific Fertility

Based on the following sets of fertility data: 1906 Taiwan [11], Standard Natural Fertility [4], 1973 Libya and 19th century Utah [6].

\section{Uncertainty about recipients}

Thus far, I have assumed that the recipient is known with certainty at the time the investment is made. No allowance has yet been made for the possibility that the benefit may eventually go to someone other than the intended recipient. As in my previous paper on time preference, I will incorporate uncertainty by assuming that when the benefit arrives, it will be allocated among potential recipients (including the donor herself) so as to maximize its discounted value to the donor. As before, I rule out the possibility of distributing the benefit among several recipients. The development below differs from that of my previous paper in two ways. First, it allows the interaction to affect fertility as well as survival. Second, it will incorporate diminishing marginal returns to consumption.

\subsection{Model}

We begin as before, with table 1 . The difference is that, under uncertainty it is not the row-sum itself that must equal zero, but the expected value of this sum. I assume changes in fertility and survival are caused by changes in consumption, as discussed above in section 2.2.2. In addition, I use the model of diminishing marginal returns defined above in section 2.3. Thus, equations 6-7 and 11-12 allow equation 5 to be re-expressed as

$$
\begin{aligned}
0=\Delta \kappa^{(1)}( & \left.\alpha m^{(1)}+\beta P^{(1)} v^{(1)}\right) / \kappa^{(1)} \\
& +\Delta \kappa^{(2)} e^{-\rho \tau} E\left\{\left(\alpha m^{(2)}+\beta P^{(2)} v^{(2)}\right) / \kappa^{(2)}\right\}
\end{aligned}
$$

where $E$ denotes the expectation. In taking this expectation, I define $v^{(2)}=0$ when there is no recipient at all. 
The MRS in fitness is obtained by rearranging this expression to obtain

$$
M R S_{F} \equiv-\frac{\Delta \kappa^{(2)}}{\Delta \kappa^{(1)}}=E\{Z\}^{-1}
$$

where

$$
Z \equiv\left(\frac{e^{-\rho \tau} r\left(\gamma m^{(2)}+(1-\gamma) P^{(2)} v^{(2)}\right)}{\left(\gamma m^{(1)}+(1-\gamma) P^{(1)} v^{(1)}\right)}\right)\left(\frac{\kappa^{(1)}}{\kappa^{(2)}}\right)
$$

and $\gamma \equiv \alpha /(\alpha+\beta)$ measures the relative importance of marginal fertility.

In what follows, I will take $\kappa^{(1)}=\kappa^{(2)}$ so that the final term in $Z$ disappears. This restricts attention to the MRS at points along the $45^{\circ}$ line in figure 1 . In intergenerational transfers there is good reason for interest in these values. At stationary equilibrium, the consumption of an individual at age $x^{(1)}$ must equal that of her daughter one generation hence. Thus, intergenrational investments are governed by the MRS in preferences along the $45^{\circ}$ line, which must also equal the MRS in exchange and the marginal productivity of intergenerational investment. ${ }^{4}$ These quantities could all be predicted from the MRS in fitness along the $45^{\circ}$ line. For transfers over shorter intervals, there is less reason for concern with the MRS along the $45^{\circ}$ line. For these cases, the present approach will tell only part of the story.

\subsubsection{The evolutionary discount function}

To facilitate presentation of numerical results, I define an evolutionary discount function $\lambda$, which satisfies

$$
M R S_{F} \equiv e^{\int_{x}^{x+\tau} \lambda(x, w) d w}
$$

For example, when $\lambda$ is a constant, future benefits are discounted exponentially at a constant rate. $\lambda$ can accomodate nearly any form of discounting, and is closely related to the marginal rate of time preference (MRTP): the average value of $\lambda$ over any age-interval predicts the MRTP over that interval [15, Eqn. 15]. I calculate $\lambda$ from age-specific fertility and survival data using the methods described by Rogers [15].

\subsubsection{Demographic statistics}

Ideally, $\lambda$ should be estimated using demographic statistics that reflect some sort of long-term average of human demographic history. This, of course, is impossible. I have instead relied on demographic statistics from modern "natural-fertility" populations, whose vital rates are thought to resemble those of pre-industrial populations. ${ }^{5}$ It would be unwise, however, to take any single modern population as the examplar of our unknown ancestors. We do not know whether prehistoric human demography was more similar to that of 19th century Taiwan, or that of 19th century Utah, to name just two possibilities. Nonetheless, it seems likely that species-wide mean demographic

\footnotetext{
${ }^{4}$ See $[13$, p. 172] and [15, Footnote 12].

${ }^{5} \mathrm{~A}$ natural-fertility population is one in which birth-control is either absent, or else is applied independently of the number of a woman's existing children. In natural-fertility populations, women may use birth control to space births, but they do not use it to achieve a target family size [1].
} 
parameters have for a very long time fallen within the range spanned by modern natural-fertility populations. In my previous paper, I estimated $\lambda$ using demographic statistics from a wide variety of natural-fertility populations, and found that this variation had little effect on the answer. Consequently, I will restrict attention here to a single set of demographic statistics. I use fertility and paternity data of 19th century Utah [6] and the Model West life table with mortality level 12 [3, p. 47]. This mortality level implies that the expectation of life at birth $e_{0}^{\circ}$ is approximately 45 years.

\subsection{Results}

Before presenting new results, I summarize some old ones. Figure 5 shows an evolutionary discount function from my earlier paper on time preference. In the figure, "age at investment" refers to the age at which a decision is made between an immediate and a delayed benefit. Ages beyond the age at investment are "future ages." Thus, the line marked by open circles shows the discount function pertaining to some investment that might be undertaken by newborn infants, whereas the line marked by stars pertains to investments by young adults.

To understand what these curves mean, consider a hypothetical 20-year old woman who has been offered some survival benefit that will not arrive until she is 40 . Since she is female and is now of age 20, the starred curve in the upper panel of figure 5 applies. It indicates that the average discount rates within the four 5-year intervals spanning ages 20-40 are 0.059, 0.050, 0.012, and 0.007 respectively. The average of these is 0.032 , and this implies ${ }^{6}$ that the future benefit should be discounted by a factor of $\exp [-20 \times 0.032]=0.529$. The 20 -year old, therefore, should value this delayed benefit at only about half of its nominal value. In general, one applies a MRTP that is an average of $\lambda$ over the relevant interval.

The figure illustrates the major conclusions of the previous analysis:

- In the long run, $\lambda$ converges to a value of about $2 \%$, very close to the value predicted by the heuristic argument leading to equation 10. This lent support to my conclusion regarding the interest rate.

- The curves for different ages of investment lie nearly atop one another. Thus, $\lambda$ is well approximated by a function of one argument: $\lambda(x, y) \approx \lambda^{*}(y)$.

- The evolutionary discount is much higher among young adults than among their elders. This predicts higher marginal rates of time preference among young adults, a prediction with which we can all identify.

However, figure 5 describes an analysis on survival axes rather than consumption axes. The evolutionary discount function there refers, in other words, to a trade-off between the survival (not the consumption) of donor and recipient. The methods introduced here allow an analysis on consumption axes, with varying levels of importance accorded to marginal fertility and marginal survival.

\footnotetext{
${ }^{6}$ The average of $\lambda$ predicts $\theta$, the MRTP. This average is equal to $\theta=0.032$, and equation 1 implies that the future benefit is discounted by a factor of $e^{-\theta \tau}$, where $\tau=20$ is the time delay.
} 

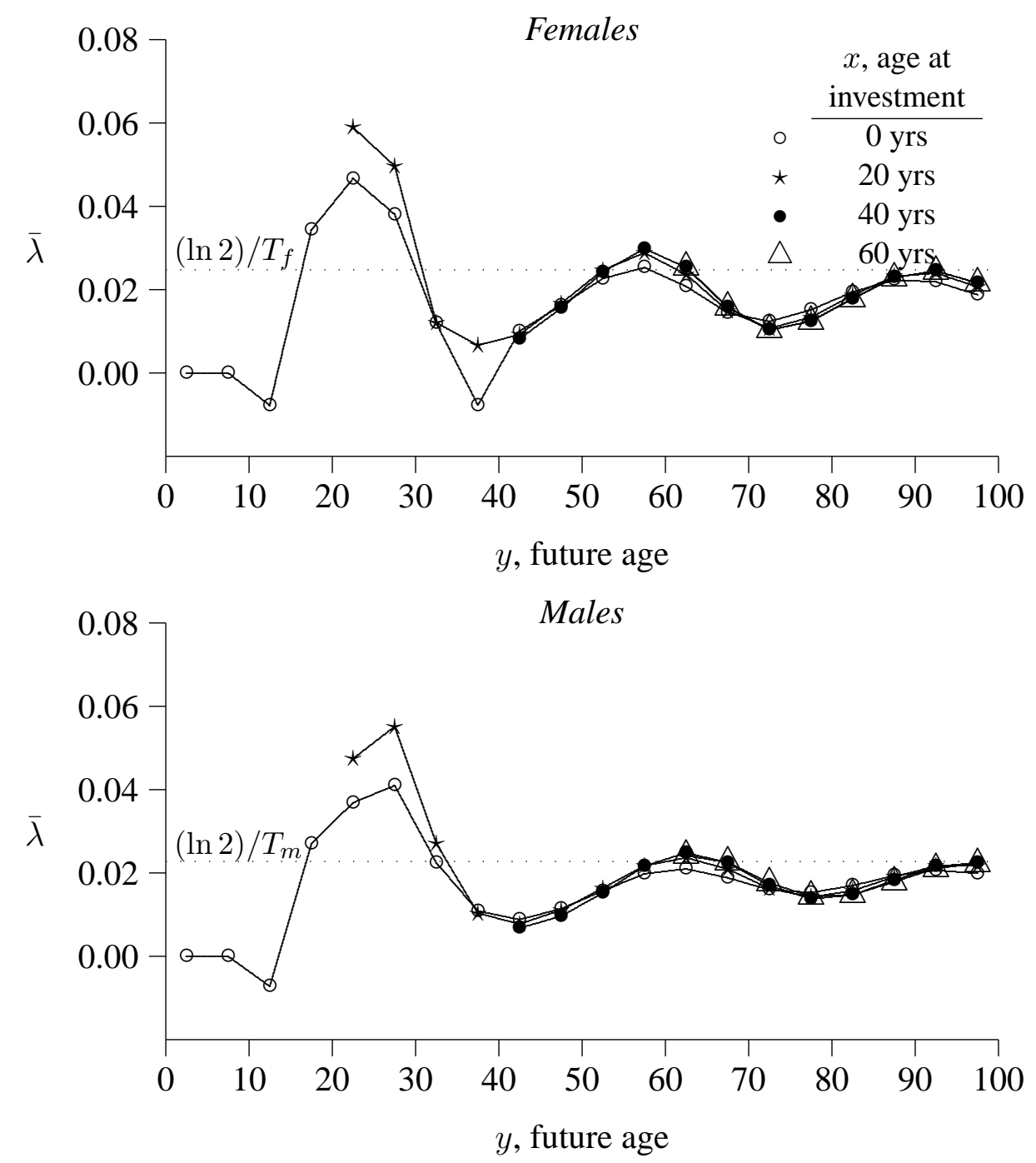

Figure 5: Evolutionary Discount Function

$\bar{\lambda}(x, y)$ is the average evolutionary discount rate within a five-year age interval. "Age at investment," $x$, refers to the age at which a decision is made between an immediate and a delayed benefit. "Future age," $y$, refers to ages beyond the age at investment. The dotted lines show the rate of interest predicted by equation 10, where the generation time is $T_{f}=27.98$ for females and $T_{m}=30.45$ for males.

Based on male and female fertility of 19th century Utah Mormons [6], and on the Model West life table (mortality level $12, e_{0}^{\circ}=47.5$ for females and 44.5 for males). 


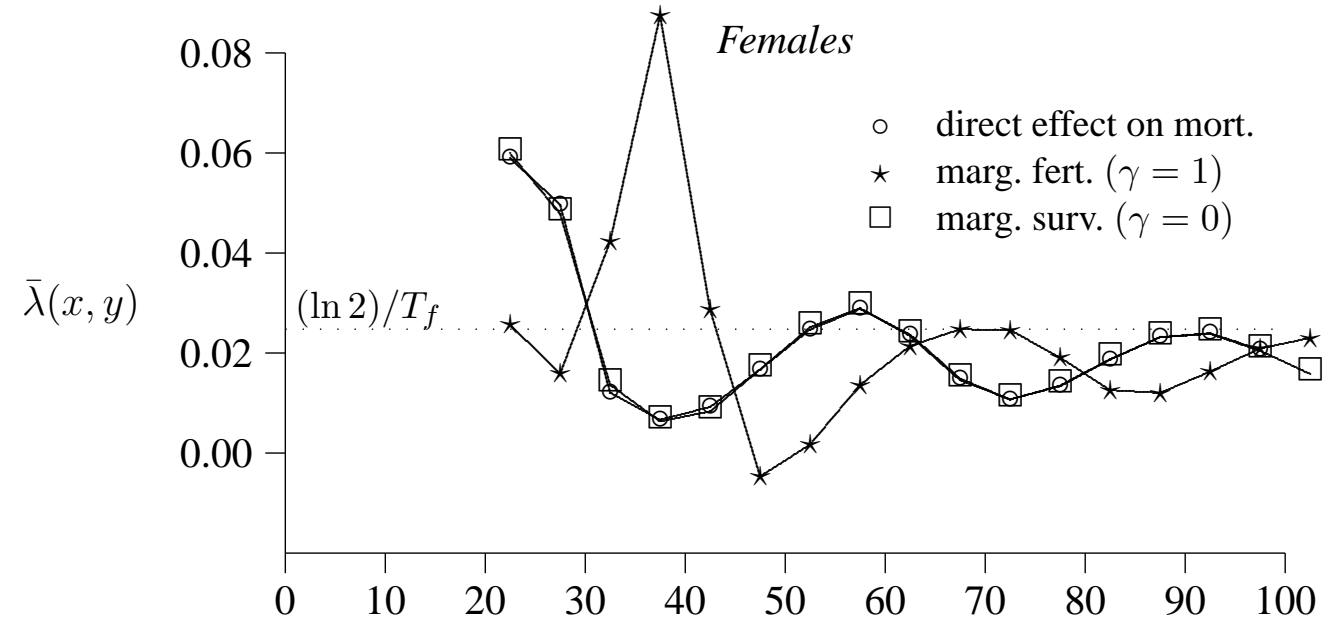

$y$, future age

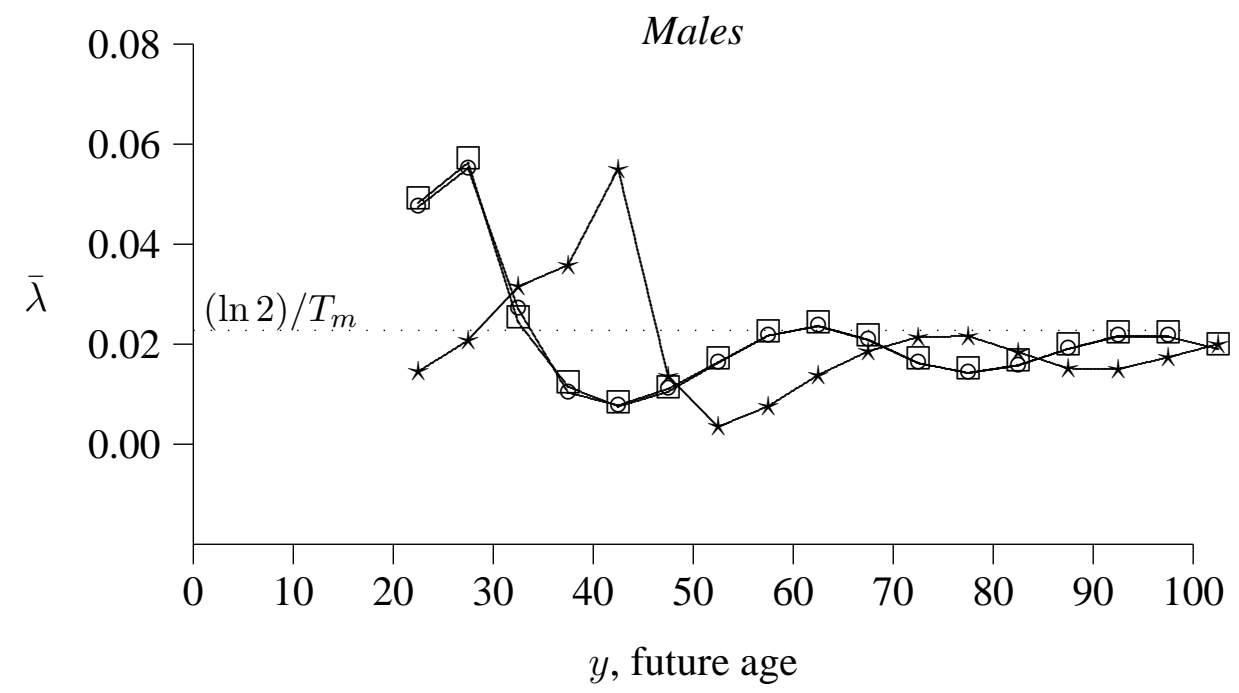

Figure 6: Evolutionary Discount Function

$\bar{\lambda}(x, y)$ is the average evolutionary discount rate within a five-year age interval. All curves refer to 20-yearold investors. "Future age," $y$, refers to ages beyond the age at investment. The dotted lines show the rate of interest predicted by equation 10 , where the generation time is $T_{f}=27.98$ for females and $T_{m}=30.45$ for males.

Based on male and female fertility of 19th century Utah Mormons [6], and on the Model West life table (mortality level $12, e_{0}^{\circ}=47.5$ for females and 44.5 for males). 
The result of this analysis is shown in figure 6 , along with the age-20 curve from figure 5 . It shows that

- The long-term tendency is toward a rate of roughly $2 \%$ in all cases. Thus, conclusions about the interest rate are unaffected by the difference between these models.

- When consumption affects survival (i.e. when $\gamma=0$ ), the curve differs little from that of the earlier analysis.

- When consumption affects fertility, the discount function peaks in the late thirties and early forties.

I'm not sure what to make of this. Perhaps:

- young people are prone to risk their lives in return for immediate gratification (fast driving, sky diving, high crime rates), but middle aged people are more prone to take risks affecting fertility.

\section{References}

[1] Elizabeth A. Cashdan. Natural fertility, birth spacing, and the "first demographic transition". American Anthropologist, 87(3):650-653, Sept. 1985.

[2] Brian Charlesworth. Evolution in Age-Structured Populations. Cambridge University Press, Cambridge, England, 1980.

[3] Ansley J. Coale and Paul Demeny. Regional Model Life Tables and Stable Populations. Academic Press, New York, 2nd edition, 1983.

[4] Ansley J. Coale and T. J. Trussell. Model fertility schedules: Variations in the age structure of childbearing in human populations. Population Index, 40(2):185-258, April 1974.

[5] James F. Crow and Motoo Kimura. An Introduction to Population Genetics Theory. Harper and Row, New York, 1970.

[6] Mahjoub A. El-Faedy and Lee L. Bean. Differential paternity in Libya. Journal of Biosocial Science, 19(4):395-403, Oct. 1987.

[7] Caleb E. Finch. Longevity, Senescence, and the Genome. University of Chicago Press, Chicago, 1990.

[8] Ronald A. Fisher. The Genetical Theory of Natural Selection. Dover, New York, 2nd edition, 1958.

[9] William D. Hamilton. The genetical evolution of social behavior, I. Journal of Theoretical Biology, 7(1):1-16, July 1964. 
[10] William D. Hamilton. The genetical evolution of social behavior, II. Journal of Theoretical Biology, 7(1):17-52, July 1964.

[11] William D. Hamilton. The moulding of senescence by natural selection. Journal of Theoretical Biology, 12(1):12-45, Sept. 1966.

[12] Ingemar Hansson and Charles Stuart. Malthusian selection of preferences. American Economic Review, 80:529-544, 1990.

[13] Jack Hirshleifer. Investment, Interest, and Capital. Prentice-Hall, Englewood Cliffs, NJ, 1970.

[14] Alan R. Rogers. Why menopause? Evolutionary Ecology, 7(4):406-420, July 1993.

[15] Alan R. Rogers. Evolution of time preference by natural selection. The American Economic Review, 84(3):460-481, June 1994. 